\title{
Wheelchair interventions, services and provision for disabled children: a mixed-method systematic review and conceptual framework
}

Nathan Bray ${ }^{1,2^{*}}$, Jane Noyes ${ }^{2}$, Rhiannon T Edwards ${ }^{1}$ and Nigel Harris ${ }^{3}$

\begin{abstract}
Background: Wheelchairs for disabled children ( $\leq 18$ years) can provide health, developmental and social benefits. World Health Organisation and United Kingdom Government reports demonstrate the need for improved access to wheelchairs both locally and internationally. The use of health economics within this field is lacking. Provision of wheelchairs based on cost-effectiveness evidence is not currently possible. We conducted the first systematic review in this field to incorporate evidence of effectiveness, service user perspectives, policy intentions and cost-effectiveness in order to develop a conceptual framework to inform future research and service development.
\end{abstract}

Methods: We used an adapted EPPI-Centre mixed-method systematic review design with narrative summary, thematic and narrative synthesis. 11 databases were searched. Studies were appraised for quality using one of seven appropriate tools. A conceptual framework was developed from synthesised evidence.

Results: 22 studies and 14 policies/guidelines were included. Powered wheelchairs appear to offer benefits in reduced need for caregiver assistance; improved communicative, personal-social and cognitive development; and improved mobility function and independent movement. From 14 months of age children can learn some degree of powered wheelchair driving competence. However, effectiveness evidence was limited and low quality. Children and parents placed emphasis on improving social skill and independence. Participation in wider society and development of meaningful relationships were key desired outcomes. Policy intentions and aspirations are in line with the perspectives of children and parents, although translation of policy recommendations into practice is lacking.

Conclusions: There is a distinct lack of high quality effectiveness and economic evidence in this field. Social and health needs should be seen as equally important when assessing the mobility needs of disabled children. Disabled children and parents placed highest priority on independence and psychosocial outcomes of wheelchair interventions. Translation of policy and guidelines into practice is lacking and more effective implementation strategies are required to improve services and outcomes. Future research should focus on outcome measure development, developing economic evaluation tools and incorporating these into high quality studies to address known research gaps. The novel conceptual framework maps current gaps in evidence and outlines areas for development.

Keywords: Wheelchair, Childhood disability, Assistive mobility technology, Children, Powered wheelchair, Systematic review, Conceptual framework, Health economics

\footnotetext{
* Correspondence: n.bray@bangor.ac.uk

${ }^{1}$ Centre for Health Economics and Medicines Evaluation, Bangor University, Ardudwy Building, Normal Site, Bangor University, Bangor, Gwynedd LL57 $2 \mathrm{PZ}, \mathrm{UK}$

${ }^{2}$ Centre for Health-Related Research, Fron Heulog, Bangor University, Bangor, Gwynedd LL57 2EF, UK

Full list of author information is available at the end of the article
}

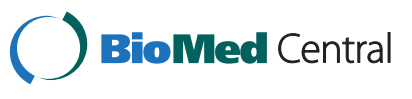

(c) 2014 Bray et al.; licensee BioMed Central Ltd. This is an Open Access article distributed under the terms of the Creative Commons Attribution License (http://creativecommons.org/licenses/by/2.0) which permits unrestricted use, distribution, and reproduction in any medium, provided the original work is properly credited. The Creative Commons Public Domain Dedication waiver (http://creativecommons.org/publicdomain/zero/1.0/) applies to the data made available in this article, unless otherwise stated. 


\section{Background}

\section{Global context}

It is estimated that between $10 \%$ [1] and 15\% [2] of the world's population is disabled. $10 \%$ of disabled people require a wheelchair to provide essential mobility assistance [3]. Approximately 20 million people worldwide do not have access to adequate wheelchairs to maintain mobility and independence, particularly in low-income countries [4]. At present there is inadequate evidence to facilitate appropriate wheelchair service provision and support for those with disabilities [2]. This relates to both understanding of intervention cost-effectiveness and estimates of disability prevalence.

Independent mobility for disabled people and provision of assistive mobility technology (such as wheelchairs) to facilitate this is considered a human right, which calls for all countries to ensure that disabled people are able to access essential assistive mobility technology to promote mobility and independence [5]. Without adequate wheelchairs many disabled people are caught in a cycle of poverty and depravation, lacking the ability to access education, work and social facilities [4]. These issues also have national economic impacts due to loss of productivity and health service resource use [2].

Approximately 5\% of children worldwide (around 95 million children aged 14 or under) have a disability [6]. Each disabled child with a mobility impairment has different needs in terms of assistive mobility technology and seating, including consideration of posture, pelvic support and head/neck support. For instance, children with cerebral palsy have the highest demand for specialised seating [7]. Wheelchairs provide essential mobility; it is imperative that they can be used in all places they are required (e.g. school, home and leisure facilities) [8] and that they support the holistic needs of each individual [9]. United Kingdom (UK) and World Health Organisation (WHO) policy states that disabling barriers must be addressed in order to limit exclusion of disabled children from education, healthcare, housing and leisure $[8,10]$.

Appropriate wheelchair interventions are therefore a global imperative in order to reduce disability discrimination and promote equality.

\section{NHS Wheelchair Services for disabled children in the United Kingdom}

There are an estimated 770,000 children and young people under the age of 16 in the UK living with a disability [10]. Several UK government and not for profit organisation (NFPO) reports have found that wheelchair services for children and young people in the UK need improvement in order to meet service user needs [11-15]. These reports reflect the need for a better understanding of the relationship between UK National
Health Service (NHS) wheelchair services, effectiveness evidence, service user perspectives and policy intentions.

\section{Why is a systematic review needed?}

Wheelchair interventions can have a range of positive impacts on the lives and health of disabled children and young people. In order to promote effective and equitable wheelchair services both in the UK and globally, better understanding of the effectiveness and cost-effectiveness of wheelchair interventions is needed. Likewise, the opinions of young wheelchair users and their families need to be taken into account to shape services. Social theories of disability state that disability exists as both a physical and social issue. Discrimination and positivist approaches to disability management pose more threat to equality than actual physical impairment [16].

Health economics can play a specific role in the development of wheelchair services by providing essential data on the cost-effectiveness of different wheelchair interventions in both developing and developed countries. This would in theory facilitate better use of resources and greater coverage of services. At present the health economics toolbox is particularly poor when applied to disabilities and children. Development of health economics methodologies based on a social model of health would promote holistic evaluation of effectiveness and costeffectiveness.

In order to develop an appropriate set of economic tools it is important to explore existing effectiveness, service user opinion and economic evidence. The development of a conceptual framework from synthesised evidence could then be used to guide wheelchair service development in an evidence-based manner. No existing systematic reviews which address these important issues were found prior to conducting this review.

To maintain clarity, we will define a number of key concepts and definitions as follows: the term "wheelchair service" is used to define any private, state or NFPO run service supplying wheelchairs to disabled people based upon assessment of mobility needs by a qualified professional. The term "wheelchair provision" is used to define the supply of a wheelchair intervention to a disabled person by a wheelchair service (as defined above). The term "wheelchair intervention" is used to define any wheelchair supplied to a disabled person by a wheelchair service (as defined above). The term "effectiveness" refers to all relevant clinical and non-clinical outcomes related to wheelchair use, such as (but not restricted to): cognitive, physical and behavioural development; functional mobility and motor skills; independence; educational achievement; social interaction; initiative development; physical and/or emotional well-being; and health-related quality of life. We do not use "effectiveness" to refer to biomechanical outcomes, such as propulsion patterns. 


\section{Aims and objectives}

The overarching aim was to explore current effectiveness evidence, service user perspectives, policy and cost-effectiveness evidence in order to develop a conceptual framework to inform future research and wheelchair service development in the UK, with international implications. Four objectives were developed to inform searching, management and interpretation of evidence:

- to determine the effectiveness and cost-effectiveness of wheelchairs for disabled children and young people

- to better understand service user, parent and professional perspectives regarding wheelchairs for disabled children and young people

- to explore current UK policy, NFPO publication and clinical guideline recommendations and intentions regarding wheelchair provision for disabled children and young people

- to determine if disabled children's desired outcomes match with existing policy aspirations and effectiveness evidence

\section{Review and synthesis questions}

Review questions were formulated for each of the different aspects of this review, with additional questions developed to guide the overarching synthesis of evidence. See Figure 1 for a full list of review questions.

\section{Methods}

\section{Design}

An initial scoping search of the literature was conducted to refine the review scope, processes and keywords. A variety of quantitative, qualitative and policy literature was found, demonstrating the multi-faceted nature of wheelchair interventions. It was therefore decided that a mixed-method systematic review would be the most appropriate way to address the issues of interest. The review questions and a protocol were then developed to guide the review. Searches were conducted between January and April 2012.

The review followed the University of York Centre for Research and Dissemination (York CRD) principles for conducting searches and extracting data [17]. A thematic synthesis approach was used to synthesise qualitative data, informed by the work of Thomas and Harden (2008) [18], while narrative summary was used to synthesise evidence within the intervention, policy and economic streams of evidence [17]. Narrative synthesis was used for the overarching synthesis of different types of evidence $[17,19]$.

An adapted Evidence for Policy and Practice Information and Co-ordinating Centre (EPPI-centre) design and methodology for mixed-method evidence [20] was used to synthesise diverse evidence. Evidence was streamed by evidence and methodology type and results were then synthesised across the streams in a final overarching synthesis (see Figure 1).

A full audit trail was recorded during each stage of the review to enable replicable methods and outcomes. During the screening process each study was screened independently by two reviewers. The second reviewer extracted data and appraised the quality of a selection of intervention studies $(n=5)$. The findings showed general consensus between the two reviewers, although full appraisal of inter-rater reliability was not conducted due to time constraints.

\section{Search methods}

Internet reference database searching was the main strategy for gathering studies. Inclusion and exclusion criteria were used to refine searches. Databases searched were Cochrane Collaboration Register and Library, Science Direct, CINAHL, Medline, ASSIA, PsychINFO, PubMed, Web of Science, DARE, NHS EED and HTA.

As wheelchair interventions have developed significantly in recent times it was deemed appropriate to restrict the intervention, opinion and economic literature searches to the last 15 years (February 1997 to February 2012). Reference list and hand-searching supplemented electronic searching. Grey literature was also included to limit publication bias. Due to limited translation resources, only studies written or translated into English (UK and international) were considered for inclusion. Search results were managed using the online bibliographic management program RefWorks.

Policy and NFPO literature was not available on academic databases. It was identified through internet search engines (Google, Google Scholar), Department of Health/relevant NFPO websites and through handsearching. Only UK policy/NFPO literature from the last 10 years (March 2002 to March 2012) was considered for inclusion to avoid obsolete literature being included in the review. Although international literature was included in the other streams, it was deemed too expansive to include all international policy in this review. Nonetheless, UK policy is evidence-based including international evidence.

Search terms and keywords were a mixture of Medical Subject Heading (MeSH) and non-MeSH terms. A full list of search terms/keywords can be seen in Table 1, and an example search strategy can be seen in Table 2. In order to ensure that all relevant studies were identified, intervention/opinion evidence search terms were divided into three groups: 'population,' 'disability' and 'intervention' (see Table 1). For the economic evidence searches an additional search term group was added: 'study type/outcomes measures'. As the aim of the mixed-method search 


\section{Review Questions}

1. What evidence is there for the effectiveness of assistive mobility technology interventions in terms of clinical, social, educational and developmental benefits for disabled children and young people (aged $\leq 18)$ ?

2. What are the perceived barriers and facilitators of providing and using assistive mobility technology for disabled children and young people (aged $\leq 18$ ), taking into account the different perspectives of disabled children/young people, parents/carers, and healthcare professionals?

3. What are the current policy, NFPO publication and clinical guideline recommendations and intentions regarding wheelchair provision for disabled children and young people (aged $\leq 18$ )?

4. What are the costs, economic implications and incremental benefits of assistive mobility technology interventions for disabled children and young people (aged $\leq 18$ )?

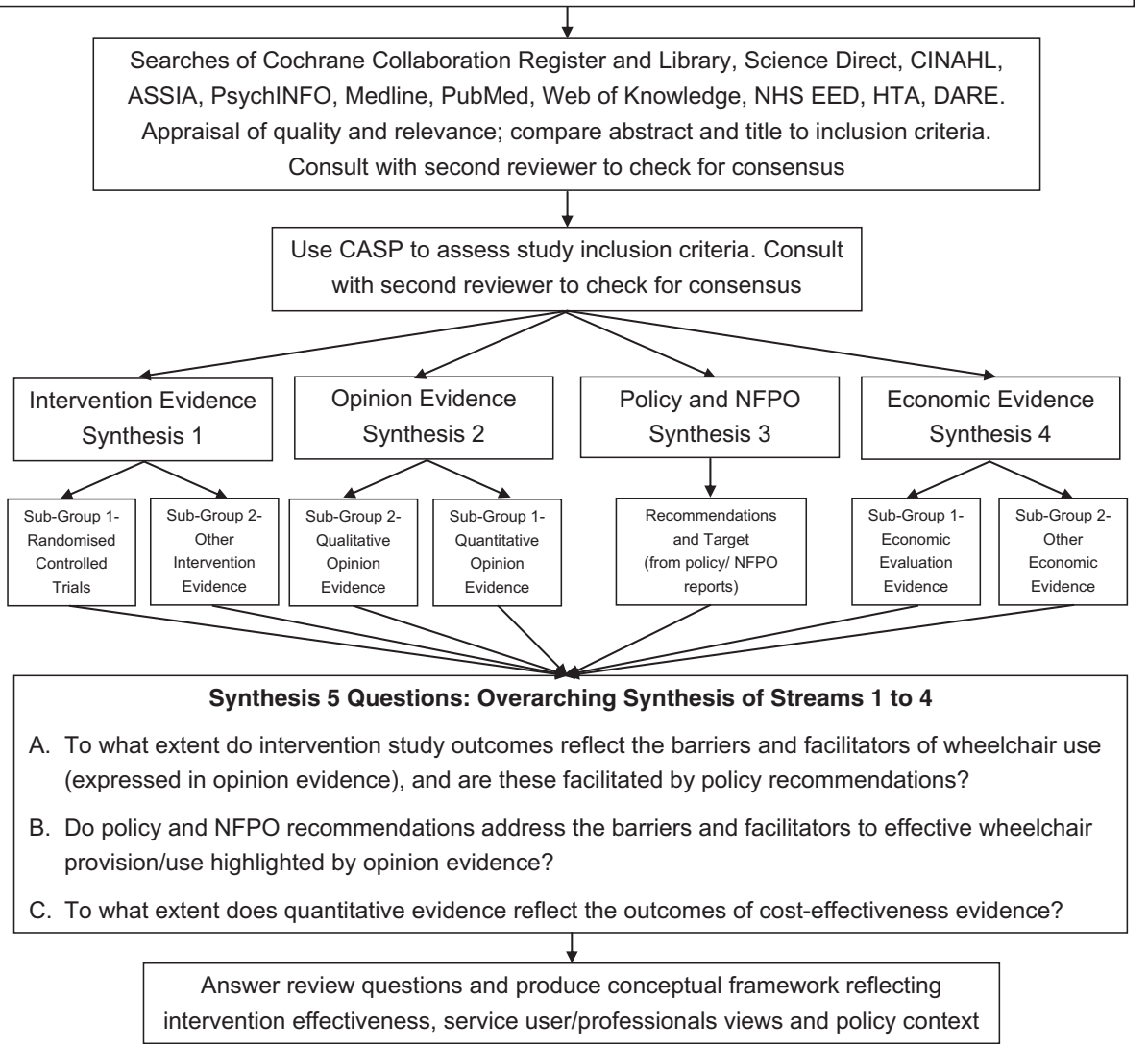

Figure 1 Systematic review design flowchart. Flowchart showing the progression of the systematic review from review questions to syntheses. Evidence was streamed by type of evidence and synthesis carried out separately for each stream. An overarching synthesis of all streams brought the different evidence together to build a conceptual framework.

was to find all relevant evidence, regardless of study type/ outcomes, it was not considered necessary to define the types of studies and outcomes eligible for inclusion. The searches were designed to be sensitive rather than specific. Testing of search terms in the initial scoping searches was used to refine search terms and to test sensitivity prior to starting the full review.

Searches focused on manual and powered wheelchairs specifically due to the volume of recent inquiries in the UK into wheelchair services, their relatively high cost and the unique benefits they provide to disabled children. Economic evidence searches were carried out separately to the intervention/opinion searches in order increase specificity. A full list of inclusion/exclusion criteria can be found in Table 3, with outcomes of interest specified.

\section{Screening}

Three stages of screening were used. For the initial screening process all identified study titles were assessed for relevance against the inclusion/exclusion criteria. A second screening process was used to assess 
Table 1 Keywords for intervention, opinion and economic evidence searches

\begin{tabular}{llll}
\hline Population & Disability & Intervention & $\begin{array}{l}\text { Study type/outcome measures } \\
\text { (economic evidence searches only) }\end{array}$ \\
\hline Child* & Disab* & Wheelchair & Cost benefit \\
Adolescen* & Physically impair* & Buggy & Cost utility \\
Young* & Physical impair* & Mobility technolog* effective* \\
Teen* & Handicap* & Mobility aid & Qaly \\
Disab* child* & Dystroph* & Powered wheelchair & Quality-adjusted life year \\
Disab* & Cerebral palsy & Mobility equipment & Quality adjusted life year \\
Adolescen* & Spina bifida & Motorised & Health economic* \\
Disab* young* & Wheelchair* & Mobility training & Economic analys* \\
Disab* teen* & Special needs & Wheelchair service & Cost minimisation \\
& Amputee & Electric scooter & Health care cost* \\
& Complex needs & Pushchair & Healthcare cost* \\
& Brain injury & Mobility & Social economic* \\
& Brain damage* & & Social care economic*
\end{tabular}

*Indicates truncation of keywords.

relevance of studies by their abstract. When relevance was unclear the full study was obtained and reviewed. All studies that were considered relevant after initial and second screening were obtained in full and underwent a final screening process. In order to reduce bias a second researcher reviewed each study independently and consensus was reached regarding inclusion. A formal screening process was not required for the policy literature, as searches were conducted using search engines and searching of government and NFPO websites. Searching stopped once saturation had been reached and no new policy/guideline reports were found.

\section{Data abstraction}

Basic information (author, publication year, title) was collected for all studies. Additionally, evidence specific data extraction tools were made for the purpose of extracting appropriate findings from the different types of literature. Each tool was tailor made for a specific type of evidence, which allowed the extraction of data to be specific to each stream of evidence. See Table 4 for a full list of data extraction criteria by evidence type.

Summary measures could not be used across the intervention evidence due to differences in sample demographics, outcome measures and interventions (see Additional file 1).

\section{Evidence synthesis}

Evidence was divided into four streams according to methodology and topic to enable separate syntheses by evidence type (see Figure 1):

1. Intervention Evidence: all quantitative studies determining the effectiveness and outcomes of relevant interventions.

2. Opinion Evidence: all studies exploring perspectives and views relating to relevant interventions in childhood disability.

Table 2 Example database search strategies

\begin{tabular}{|c|c|}
\hline Database & Search strategy \\
\hline CINAHL and MEDLINE & $\begin{array}{l}\text { Abstract only, 1997-2012 AB ( child* OR adolescen* OR young* OR teen* ) AND AB ( disab* OR physically } \\
\text { impair* OR physical impair* OR handicap* OR dystroph* OR cerebral palsy OR spina bifida OR wheelchair* } \\
\text { OR special needs OR amputee OR complex needs OR brain injury OR brain damage* ) AND AB ( wheelchair } \\
\text { OR buggy OR mobility technolog* OR mobility aid OR powered wheelchair OR mobility equipment OR motorised } \\
\text { OR mobility training OR wheelchair service OR electric scooter OR pushchair OR mobility NOT crutch* NOT prosthe*) }\end{array}$ \\
\hline ASSIA & $\begin{array}{l}\text { 1997-2012 all(child* OR adolescen* OR young* OR teen*) AND all(disab* OR physically impair* OR physical impair* } \\
\text { OR handicap* OR dystrophy* OR cerebral palsy OR spina bifida OR wheelchair* OR special needs OR amputee OR } \\
\text { complex needs OR brain injury OR brain damage*) AND all(wheelchair OR buggy OR mobility technology* OR } \\
\text { mobility aid OR powered wheelchair OR mobility equipment OR motorised OR mobility training OR wheelchair } \\
\text { service OR electric scooter OR pushchair OR mobility) AND all(cost benefit OR cost utility OR cost effective* OR } \\
\text { qaly OR quality-adjusted life year OR quality adjusted life year OR health economic* OR economic analys* OR } \\
\text { cost minimisation OR health care cost* OR healthcare cost* OR social economic* OR social care economic*) }\end{array}$ \\
\hline
\end{tabular}


Table 3 Study inclusion and exclusion criteria (by review question)

\begin{tabular}{ll}
\hline Review question & Inclusion \\
\hline 1 & Participants: Aged 18 or under with a long-term need for mobility
\end{tabular}

equipment for management of a physical disability

Interventions: Powered (independent or parent controlled) and manual wheelchairs, buggies and pushchairs

Outcomes: All relevant clinical and non-clinical outcomes, including (but not restricted to) improved cognitive, physical or behavioural development, improved motor skills, independence, educational achievement, social interaction, initiative development, physical and/or emotional wellbeing and health-related quality of life

Evidence: All effectiveness evidence related to effectiveness of assistive mobility technology including randomised controlled trials, quasi-experimental trials, clinical trials, epidemiological research, cohort studies, non-randomised controlled trials, mixed-method research, systematic reviews and survey data.

Outcomes: All experiences, views, perspectives, thoughts and feelings
Participants: Children/young people aged 18 or under with a long-term need for mobility equipment for management of physical disability, parent/carer of a child or young person aged 18 or under with a long-term need for mobility equipment for management of a physical disability, healthcare professionals treating/rehabilitating children/young people aged 18 or under with a long-term need for mobility equipment for management of a physical disability

Interventions: Powered (independent or parent controlled) and manual wheelchairs, buggies and pushchairs of children/young people, parents and healthcare professionals towards mobility equipment and provision

Evidence: All studies using qualitative methodologies, including ethnographic research, grounded theory research, case studies, phenomenological research, qualitative systematic reviews, meta-ethnography, mixed-method research and survey data.

'Audience: Children/young people aged 18 or under with a long-term need for mobility equipment for management of physical disability, parent/carer of a child or young person aged 18 or under with a long-term need for mobility equipment for management of a physical disability, healthcare professionals treating/rehabilitating children/young people aged 18 or under with a long-term need for mobility equipment for management of a physical disability, decision and policymakers influencing NHS wheelchair services

Publications: All policy, guidelines, frameworks and government and third sector publications regarding mobility equipment provision, use, maintenance and funding

Participants: Aged 18 or under with a long-term need for mobility equipment for management of a physical disability

Interventions: Powered (independent or parent controlled) and manual wheelchairs, buggies and pushchairs

Outcomes: All relevant clinical and non-clinical outcomes, including (but not restricted to) improved cognitive, physical or behavioural development, improved motor skills, independence, educational achievement, social interaction, initiative development, physical and/or emotional well-being and health-related quality of life. Direct and indirect costs, impacts on quality-adjusted life years gained, utility scores, quality of life measures and incremental cost-effectiveness will inform the economic outcomes.

Evidence: All economic evidence related to assistive mobility technology including cost-benefit, cost-utility and cost-effectiveness analyses. Partial economic evaluations (including cost analyses, cost-description studies and cost-outcome descriptions) will also be included. Economic evaluations conducted alongside RCTs, quasi-experimental trials, clinical trials, epidemiological research, cohort studies and non-randomised controlled trials will all be considered

\section{Exclusion}

Participants: aged over 18, short-term need for mobility equipment (e.g. wheelchair after leg fracture)

Interventions: crutches/sticks, walking frames, adapted shoes, callipers and prostheses, adaptive seating

Outcomes: All outcomes not stated in inclusion criteria

Paper details: Not written or translated into English, published over 15 years ago

Participants: children/young people and parents/ carers/healthcare professionals of people aged over 18 , short-term need for mobility equipment (e.g. wheelchair after leg fracture)

Interventions: crutches/sticks, walking frames, adapted shoes, callipers \& prostheses

Outcomes: All outcomes unrelated to barriers, facilitators, positives and negatives of mobility equipment provision

Paper details: Not written or translated into English, published over 15 years ago

Audience: children/young people and parents/carers/ healthcare professionals of people aged over 18, service users with short-term need for mobility equipment (e.g. wheelchair after leg fracture)

Publications: Policy and guidelines from outside of United Kingdom, Obsolete or out-of-date policies and guideline, published over 10 years ago

Participants: aged over 18, short-term need for mobility equipment (e.g. wheelchair after leg fracture)

Interventions: crutches/sticks, walking frames, adapted shoes, callipers and prostheses

Outcomes: All outcomes not stated in inclusion criteria

Paper details: Not written or translated into English, published over 15 years ago 
Table 4 Data extraction criteria by evidence type

Intervention evidence

Opinion evidence

Policy/NFPO literature

Economic evidence
Aims, objectives, hypotheses, study type, methodology, randomisation details, number of groups, number in each group, number completed in each group, data collection time points, participant characteristics, participant age range, type of intervention(s), inclusion/exclusion criteria, country/ethnicity, baseline characteristics, content of intervention(s), duration of intervention(s), control intervention(s), follow-up period, outcomes and measures, narrative summary of findings (including statistical significance, confidence intervals and effect size), identified themes/concepts.

Aims, objectives, hypotheses, study type, methodology, number of study groups, number in each group, number completed in each group, data collection time points, participant characteristics, participant age range, type of intervention(s), inclusion/exclusion criteria, country/ethnicity, follow-up period, narrative summary of findings, identified themes/concepts.

Type of publication, topic, aims, objectives, related conditions and disabilities, age range of affected individuals/target audience, related interventions, narrative summary of recommendations and guidance.

Perspective, aims, objectives, hypotheses, study type/methodology, price year/currency, randomisation details, number of groups, number in each group, number completed in each group, data collection time points, measure of benefit, participant characteristics, participant age range, type of intervention(s), inclusion/exclusion criteria, country/ethnicity, baseline characteristics, content of intervention(s),

duration of intervention(s), control intervention(s), follow-up period, outcomes and measures, narrative summary of findings (including statistical significance, confidence intervals and effect size), identified economic costs and implications, cost per QALY/Incremental cost-effectiveness ratio conclusions, inflated (2012) cost per QALY/Incremental cost-effectiveness ratio conclusions.
3. Policy and NFPO Literature: all relevant policy, NFPO and clinical guideline literature.

4. Economic Evidence: all relevant economic and cost-effectiveness evidence.

Intervention and economic streams were not synthesised due to vast differences in studies and lack of statistical evidence within each stream (see Additional file 1 for further details), thus narrative summary was conducted. Intervention evidence outcomes were grouped by type.

For the qualitative opinion evidence, thematic synthesis [18] was conducted in order to identify key themes of service user and professional perspectives on wheelchair provision and interventions. This process included three stages:

1. Line-by-line coding of findings to order the findings into initial codes

2. Grouping of initial codes to form broader descriptive themes

3. An overarching synthesis of the descriptive themes to create higher-level analytical themes.

Survey data that could be coded (such as open-ended questions) was incorporated into the thematic synthesis. For survey evidence that could not be line-by-line coded, narrative summary was used to form a structured narrative of results. These data were later synthesised with the thematic synthesis findings and incorporated into the appropriate descriptive themes.

A final over-arching narrative synthesis was undertaken to draw together the results across the different streams of evidence. The framework developed by Oliver et al. [19] was used to structure this synthesis and compare results across streams of evidence. To facilitate this three overarching questions were developed (see Figure 1).

\section{Conceptual framework development}

A conceptual framework for developing cost-effective wheelchair services for children and young people was refined from the overarching synthesis of evidence. Findings from the different streams of evidence were interrogated, discussed, mapped, charted and refined through further discussion within the research team to build a deeper understanding. The most important findings were selected and integrated into a conceptual diagram. A programme theory for an evidence-based pathway through wheelchair services was developed which highlighted gaps in knowledge and current services.

\section{Results}

\section{Search and screening outcomes}

A full list of included studies can be found in Additional file 1. See Figures 2 and 3 for the screening process outcomes. In total 4144 studies were found in the intervention/opinion evidence searches, of which 2393 duplicates were removed (see Figure 2). After screening titles and abstracts, 76 full-texts were left. In total a further 56 were excluded after screening of full-texts, leaving 20 deemed eligible for inclusion: 10 in the intervention evidence stream and 14 in the opinion evidence stream (four studies were eligible for both streams of evidence). Reasons for exclusion included focus on adults (or inability to extract child data), lack of primary data and focus on biomechanical outcomes.

In total 389 studies were found in the economic evidence searches, of which 163 duplicates were removed (see Figure 3). After screening titles and abstracts, seven 


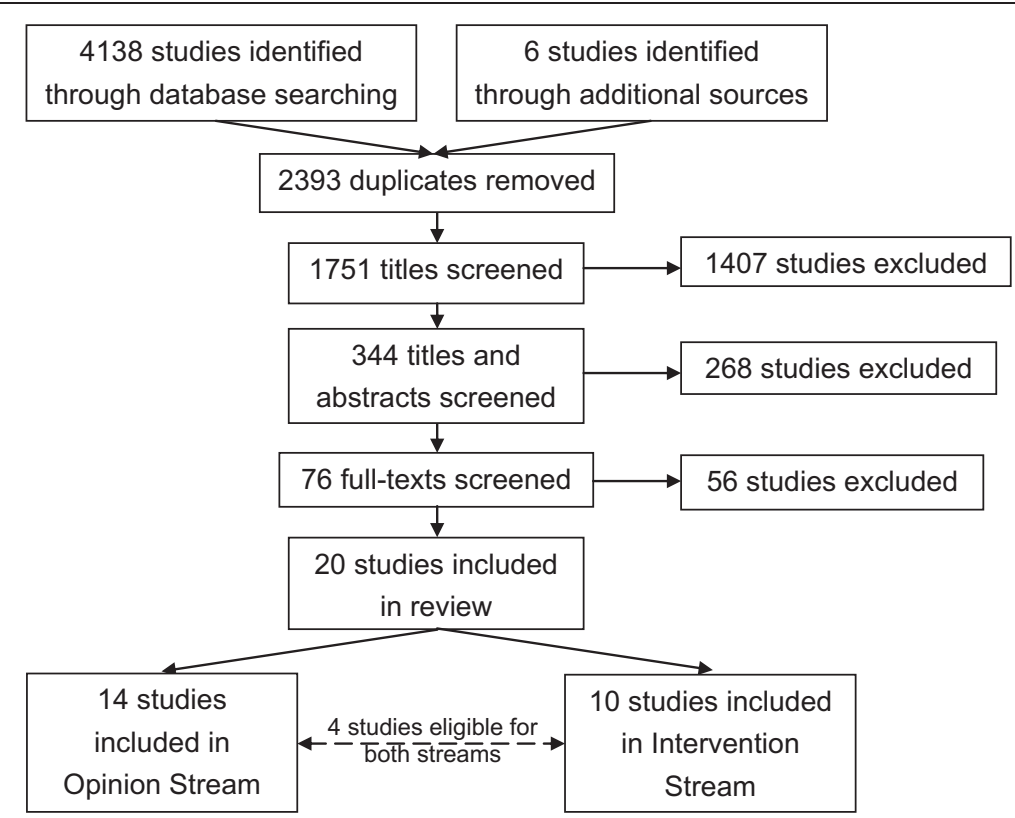

Figure 2 Search results for intervention and opinion evidence searches. Flowchart showing the search results and screening stages of the intervention/opinion evidence searches.

full-texts were left. In total two were deemed eligible for inclusion. Reasons for exclusion included focus on adults and lack of primary data. In total 14 policy and NFPO reports were deemed eligible for inclusion.

Evidence could not be grouped and analysed using meta-analysis due to heterogeneity of samples, methodology, interventions and outcomes (see Additional file 1). Summary outcomes and synthesis of statistical data were also inappropriate due to heterogeneity. Narrative summary was conducted to form a structured narrative of the results.

\section{Intervention evidence}

10 studies explored the effectiveness of wheelchairs for children with disabilities: Seven determined the effectiveness of powered wheelchairs (PWC) [21-27]; one compared ultralight and lightweight wheelchairs [28]; and two looked generally at the impact of assistive devices/environmental modifications (including PWCs) [29,30].

Of the 10 studies, five looked specifically at children with cerebral palsy and orthopaedic disabilities [22,24,25,27,29]. The remainder included children with a range of disabilities such as complex developmental delay [26],

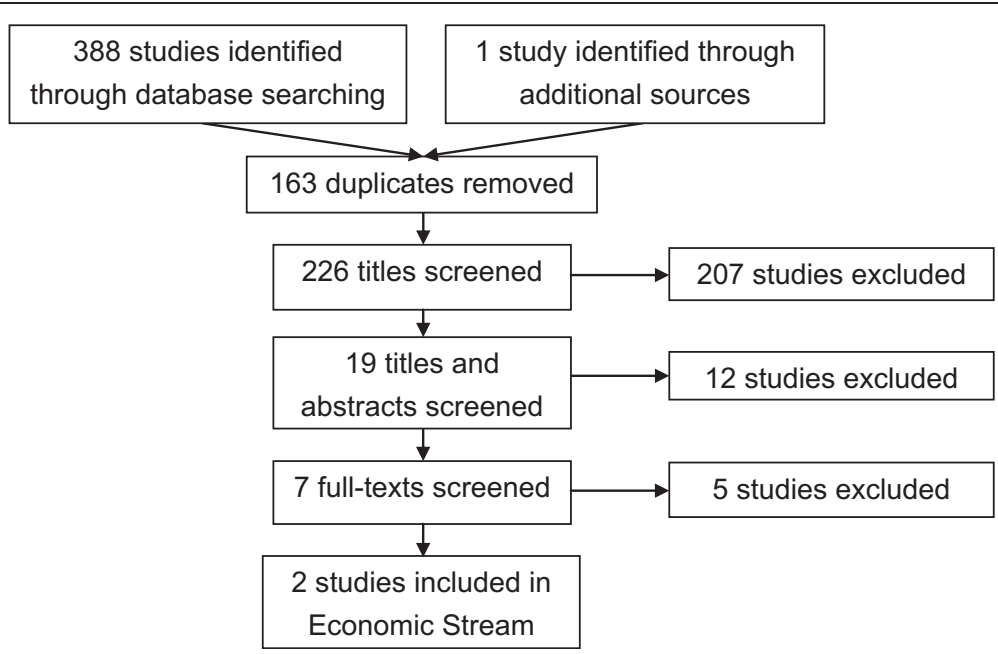

Figure 3 Search results for economic evidence. Flowchart showing the search results and screening stages of the economic evidence searches. 
spinal muscular atrophy [21], spina bifida [28] and motor impairment preventing functional independent mobility (including conditions such as cerebral palsy) $[23,30]$. Child participant ages ranged from 14 months to 12 years.

Only one randomised controlled trial (RCT) was found [23]. The remaining studies used a range of methodologies, including case study, case series, quasi-experimental design, 'A-B-A' single subject design, single-subject withdrawal design and cross-sectional survey.

Only four studies employed statistical analysis and sample sizes were small; several studies used a single casestudy design. The single RCT [23] was of moderate quality and had a small sample size of 28 (equally split between intervention and control groups). Outcome measures used within each study are presented in Additional file 1.

Quality appraisal of studies indicated that they were generally low quality (see Additional file 2). Risk of bias was assessed as part of the critical appraisal outcomes (see Additional file 2). The intervention evidence results are therefore presented with caution, taking into account the quality of results and potential risk of bias.

The vast majority of the evidence was in reference to PWCs, and thus wider understanding of wheelchair effectiveness in general is not possible. There was some evidence to indicate that ultralight wheelchairs are preferable to lightweight chairs in terms of ease of propulsion [28].

The intervention findings were grouped by type of benefit and categorised accordingly through narrative summary. Statistical significance is presented where reported. The emergent categories of benefit were:

\section{Caregiver assistance and benefits}

PWCs reduce need for caregiver assistance [21,23,29] and reduce caregiver stress [24]. PWCs have statistically significant effects on need for caregiver assistance for mobility ( $\mathrm{p}=.01, \mathrm{ES}=12.35[6.5-20.5]$ at $90 \% \mathrm{CI})$ and self-care $(\mathrm{p}=.0007, \mathrm{ES}=11.95[7.5-16.15]$ at $90 \% \mathrm{CI})[23]$.

\section{Social and play skills}

For children with orthopaedic disabilities aged 18 to 72 months PWCs significantly positively affect: pro-social adaptive social behaviour $(\mathrm{F}=5.30, \mathrm{p}<.05$ at $95 \%$ Confidence Interval $[\mathrm{CI}])$; interactions with family $(\mathrm{F}=3.2$, $\mathrm{p}<.05$ at $95 \% \mathrm{CI})$; indoor play motor activities $(\mathrm{F}=4.53$, $\mathrm{p}<.05$ at $95 \% \mathrm{CI})$; quality of interactive play $(\mathrm{F}=4.24$, $\mathrm{p}<.05$ at $95 \% \mathrm{CI}$ ); and developmental level of symbolic play $(\mathrm{F}=4.9, \mathrm{p}<.05$ at $95 \% \mathrm{CI})[22]$.

For children with orthopaedic disabilities aged 18 to 42 months PWCs also facilitate significant improvements in: interactions with family $(\mathrm{F}[2,21]=3.3, \mathrm{p}<.05)$; parental satisfaction with child's social and play skills $(\mathrm{F}[2,21]=$ $3.27, \mathrm{p}<.05)$; and parents' belief that the general public accepts their child $(\mathrm{F}[2,21]=3.65, \mathrm{p}<.04)[24]$.

\section{Functional movement and mobility}

PWCs improve functional mobility $[21,23]$ and childinitiated movement [26], with significant impacts on mobility functional skill $(\mathrm{p}=.04, \mathrm{ES}=6.5[2-11]$ at $90 \% \mathrm{CI})$ [23] and parental satisfaction with child's ability to go where they desire $(\mathrm{F}[2,21]=11.69, \mathrm{p}<.05)[24]$.

\section{Developmental benefits}

PWCs potentially offer developmental benefits in: communication, cognition and personal-social domains [21]; receptive communication skills [23]; and occupational performance [25]. PWCs can significantly improve: activities of daily life (in the dimension of functional limitation) $(\mathrm{p}<0.00001)$ [25]; receptive communication ( $\mathrm{p}=.03$, Effect size $=6.1[0.95-9.2]$ at $90 \% \mathrm{CI})$ [23]; and overall development scores $(\mathrm{p}=.083, \mathrm{ES}=2.0[0.0-3.5]$ at $90 \% \mathrm{CI})[23]$.

\section{Driving skill and competence}

Children as young as 14 months can learn some degree of PWC driving competence [27]. PWC driving competence improves after six to eight months of use $(\mathrm{p}<0.01)$ for children with cerebral palsy aged three to eight years [25].

\section{Opinion evidence}

14 studies explored the experiences and perspectives of young wheelchair users, their parents/carers and related professionals (e.g. clinicians, teachers, therapists). Seven studies were related specifically to PWCs $[24,31-36]$ and six were related to both manual wheelchairs and PWCs [29,30,37-40]. The majority of studies explored physical disabilities generally in children using wheelchairs (manual and/or powered), although four of the studies looked specifically at children with cerebral palsy and orthopaedic disabilities [24,29,38,39].

Most of the participants were families of disabled children (child age range from 18 months to 18 years), although four studies also included professional participants (e.g. wheelchair suppliers, teaching staff, therapists, clinicians) [32,33,35,39] and four directly included the opinions of disabled children and young people [36-38,40]. Five studies used qualitative methodologies exclusively (including phenomenology and grounded theory) $[31,32,37,38,40]$, while the rest used either questionnaire/ telephone survey (with quantitative and qualitative data), retrospective research or cross-sectional research. 
12 descriptive themes were generated from line-byline coding of the evidence (see Table 5), which were then synthesised to make higher-order analytical themes. Analytical theme generation was focussed on PWCs due to the focus of the qualitative evidence. Making broader assumptions about other forms of assistive mobility technology (e.g. manual wheelchairs and pushchairs) would have been inappropriate due to the lack of evidence. In total, five analytical themes were developed:

1. Wheelchair services do not consistently meet all needs of service users, and parents are resigned to this

Specific wheelchair service issues included long waiting times [37,38], poor maintenance procedures [34,37,38], strict eligibility criteria [34] and differing opinions of needs $[31,36]$. There appeared to be consensus that services were doing the best they could, and thus there was resignation to current standards of provision [37].

Participants highlighted issues around lack of information provision with regards to choice of wheelchairs, potential wheelchair benefits and funding available to families $[34,38,39]$. The evidence highlighted the financial burden placed on families having to pay for their own essential wheelchairs, maintenance and adaptations [34,36,38].

2. Parents find it difficult to accept their child's need for a wheelchair

There was a perception that accepting a wheelchair was an admission that independent mobility without a wheelchair would never be possible [31]. The process of coming to terms with both manual wheelchair and PWC use for their children was long, and for many parents

Table 5 Descriptive themes generated from opinion evidence

\begin{tabular}{ll}
\hline Descriptive themes & Examples \\
\hline Wheelchair services & Providers, repair and maintenance \\
Environmental factors & Home, public and school environment \\
Chair characteristics & Size, weight and usability \\
Individual ability & Health, physical and developmental \\
readiness \\
Family factors & Attitude, support and finances \\
Learning to use wheelchair & Build quality, accidents and safe use \\
Social factors & Learning mobility and wheelchair safety \\
Quality of Life & Socialisation, participation and others' \\
Physical factors & attitudes \\
Independence & Self-esteem, confidence and well-being \\
Developmental impact & Comfort, support and positioning \\
\hline
\end{tabular}

the perception of wheelchairs was negative before their child had used one [31,33]. Results indicated that $84 \%$ (of $\mathrm{n}=25$ ) of parents did not accept the idea of PWC before provision, but $92 \%$ (of $n=25$ ) had positive feelings after PWC provision [17]. This demonstrates that a process of adjustment is required. 23\% (of $n=140$ ) of wheelchair clinicians and suppliers felt that a lack of family support limited wheelchair provision [33].

\section{PWCs are a tool for independence and socialisation}

PWC use facilitates development of independence in disabled children $[25,31,34,38]$, and this independence subsequently allows greater socialisation [37]. It was found that the use of a PWC had a positive effect on the attitudes of others $[31,34]$ with people seeing the child as an independent 'whole person' [31]. This change in the attitudes of others allowed further socialisation, ageappropriate activities and acceptance by peers and other people in the community [31].

4. Wheelchairs offer a new lifestyle to disabled children and their families

Wheelchairs were perceived to offer a new lifestyle for disabled children and their families [25,30,31,34,37]. PWCs were believed to provide improvements to quality of life (over no wheelchair equipment and manual wheelchairs) [24,34]; ability to take part in age-appropriate activities and responsibilities [31,37]; and overall freedom [36]. After PWC provision children were able to socialise more [31,34,36-38]; to integrate better into school and community settings [37]; and were less reliant on the help of others [31]. Parents acting as 'responsive partners' facilitate learning to use a PWC [32].

5. Structural and environmental factors are a major barrier to the use of wheelchairs

Poor access to buildings [29,31,36,38], difficulty transporting wheelchairs $[24,29,31,33,36,37,39]$ and poor disabled parking facilities [36,38] were identified barriers to wheelchair use. Community and social environments were reported to often be unfit for wheelchair access [31,36-38]. The size and bulk of wheelchairs was reported to inhibit integration with other peers as well as affecting use and transport $[38,40]$.

\section{Policy and guidelines}

14 policy and NFPO reports were included in the review: three were produced by NFPOs [41-43]; 10 were produced by UK government and Department of Health organisations [8,9,11-15,44-46]; and one was a joint publication produced by the UK government and an NFPO [47]. 
Findings from the policy and NFPO evidence were grouped by type of recommendation/target. Seven emergent categories were identified:

\section{Waiting times}

The most commonly identified recommendation was reduction of waiting times for assessment, delivery and maintenance of wheelchairs (e.g. maximum of 18 weeks from referral to delivery $[41])[8,9,12-15,41,43,44]$.

\section{Joint-working and multi-agency approach}

The need for joined-up working between health, social care, education and NFPOs was a recurrent theme throughout the literature, with a general aim to improve services and to extend the scope of provision $[8,9,11,12,14,15,45]$. This included pooling of budgets [11] and outsourcing training/tuition [9].

\section{Effective use and outcomes}

Several publications highlighted the need for wheelchairs to be useable in all places required in order to maximise effectiveness $[8,12,15]$. There were recommendations for assessment and provision to take into account the holistic needs of service users as part of maximising social, physical and lifestyle outcomes and promoting independence $[9,11,12,15,41]$.

\section{Funding and procurement}

Recommendations included: ring-fenced budgeting for PWC provision [41]; improved efficiency, productivity and innovations in the NHS wheelchair product line [9];pooling of budgets between health, social care and education authorities [11]; and efficient procurement, long-term cost control and initial investment [47]. Productivity savings should be re-invested into wheelchair and seating provision [9].

\section{Aftercare and information}

Maintenance and review procedures need attention, with clear and defined minimum standards for reviews $[8,13,15]$. Better quality information for service users regarding support, additional funding/grants, tuition and local service changes was recommended $[8,14]$.

\section{Eligibility criteria and assessment}

Comprehensive access to multi-disciplinary assessments was of high priority $[14,15,43,45]$. There was also recommendation for extended equipment loan programmes
[45] and national consensus of eligibility criteria and outcomes $[13,46]$.

\section{Service user involvement}

Recommendations included: designing services around the needs of service users $[9,43,45]$; supporting service users to make informed decisions about treatment/care and support $[15,44]$; and improving communication with users and stakeholders [11].

\section{Economic evidence}

Two eligible studies exploring the cost-effectiveness of wheelchairs for disabled children were found. Due to the lack of evidence and the heterogeneity of data (cost, year, outcomes, interventions etc.) it was not possible to synthesise the findings, therefore narrative summary was conducted.

Neilson et al. [48] found the cost per quality-adjusted life year (QALY) (compared with a 'do nothing' scenario) for provision of a powered indoor/outdoor wheelchair ranged from $£ 734$ to $£ 1378$ (dependent on time horizon) based on a cost per wheelchair intervention ranging from $£ 1500$ to $£ 2000$. Inflation to 2011 prices $[49,50]$ provides a cost per QALY of $£ 1187$ and $£ 2229$ (40 and 50 year time horizon respectively). These results indicate that PWC interventions can be cost-effective in relation to the National Institute for Health and Clinical Excellence (NICE) $£ 20,000$ to $£ 30,000$ intervention cost threshold. Estimates are based on a single subject within the study, whose age is not stated. Costs used to generate QALYs were based on a single intervention over a 40 or 50 year time horizon.

Frontier Economics [51] examined the impact of NFPO (Whizz-Kidz) involvement in the running of NHS Primary Care Trust paediatric wheelchair services. Meeting unmet service demands cost an extra $£ 108,000$ and provided an additional 10.7 to 14 QALYs, resulting in a cost per QALY of between $£ 7,700$ and $£ 9,800$ for meeting unmet service demands. This evidence has not been published by a peer reviewed journal, thus its application in this review is limited.

\section{Over-arching synthesis}

The majority of data were specifically about PWC provision and use, which is reflected in the over-arching synthesis. A number of additional findings were elicited from further synthesis of the entire integrated dataset:

\section{Higher quality wheelchair services take into account the needs of the whole family}

Intervention and opinion evidence shows that wheelchair provision can be beneficial for both the wheelchair user and their family, including parental independence [30,37]; reduced need for caregiver assistance [21,29,30]; 
facilitation of positive parental feelings [25]; and reduction in parental stress [24].

As use of a PWC requires family involvement it is important that the home environment and the ability to transport a wheelchair is assessed and facilitated where possible. The cost of maintenance, repairs and adaptations can be prohibitive for families [36,38], thus funding arrangements at policy level should ensure that these costs are covered or available grants are signposted $[8,41]$.

Each service user may benefit from having a clear point of contact for any queries they may have $[8,13,15]$. Services may be best developed in consultation with children and families to promote child and family-centred services $[9,44]$.

\section{Disabled children benefit when psychosocial needs are considered alongside health needs}

The psychosocial needs of children using PWCs appear to be of highest priority for service users and their parents [31,34,36-38]. Children are perceived to benefit more when PWC provision takes into account where the wheelchair will be used, and ensures that any supplied PWC is fit for use in all places it is required $[8,12,15]$. Social benefits of PWCs were found in the intervention evidence, including positive differences in interactions with family after PWC intervention [24] and pro-social adaptive social behaviour [22]. A holistic approach to assessment, with performance measures that consider psychosocial, environmental and lifestyle needs alongside clinical requirements are therefore important $[9,11]$. Additional benefits and efficiencies were also noted from joined-up working and planning between health, social services and education departments $[8,9,11,12,14,15,45]$.

It is of note that the majority of opinion evidence $(n=9)$ related to children aged under 14 years. This indicates that there may be a lack of evidence on key periods of transition, such as moving from child to adult services.

\section{Disabled children could benefit if policy recommendations focussed on services meeting individual needs rather than following strict eligibility criteria}

Inefficiencies (such as long waiting times) need to be reduced $[11,37,38]$ and loan programmes developed to allow children to try wheelchairs before provision [33]. Strict eligibility criteria can be prohibitive to each child receiving the right wheelchair $[12,15,34]$, thus uniform and flexible national eligibility criteria may help to address inequity in services $[13,41,46]$. Joined-up working between agencies could further enhance services $[8,9,11,12,14,15,45]$.

Without appropriate outcome measures the holistic benefits of PWC interventions cannot be evaluated

Evidence of effectiveness and validated clinical practice outcome measures are needed in all aspects of health services [52]. The development of reliable and valid measures of holistic benefits is needed in order to measure the wider benefits of PWC interventions. When appropriate outcome measures are available PWC intervention goals can more easily focus on the tangible benefits of developmental gains rather than just development.

Opinion evidence continually demonstrated the importance of independence and the subsequent perceived benefits to service users and families [25,31,34,38]. Developmental benefits were observed in intervention and opinion evidence $[21,23,31,32,38]$. Opinion evidence highlighted the potential quality of life benefits of PWCs, including increased happiness, enjoyment of life, motivation, self-confidence and reduced frustration [34], as well as increased dignity and activities of daily living [31].

\section{Disabled children may benefit more when physical outcomes of PWC use are seen as facilitators to wider holistic benefits, but lack of translation of evidence into practice hinders progress}

The key benefits from provision of wheelchairs for service users and their families were lifestyle oriented, with a focus on social and independence effects. Policy and NFPO literature does take into account these wider benefits; recommendations highlight the need to set minimum standards for wheelchairs that are useable in all places required $[8,12,15]$ and that promote independence $[12,41]$ with measurable outcomes. However the translation of these recommendations into practice is apparently weak.

\section{Disabled children would benefit from public buildings and spaces that promote inclusion of disabled people}

Policy and NFPO literature states that wheelchairs should be useable in all places they are required $[8,12,15]$, however this appears to be in reference to the wheelchair itself rather than more accessible public places. Poorly designed public spaces restrict children's ability to participate socially $[29,31,36-38,40]$. Legally enforced equality of access is therefore likely to improve wider lifestyle benefits of wheelchair users.

Home adaptation with clear advice provided to all service users and families regarding funding and grant entitlements are also important factors that impact on health-related quality of life outcomes [8]. Some families have issues using wheelchairs due to inaccessibility of the home environment $[33,39]$. Regular review and maintenance procedures can help to ensure that wheelchairs are fit for purpose [13,41].

\section{Conceptual framework}

The conceptual framework (see Figure 4) maps how further research and service development can lead to cost-effective wheelchair services and interventions. It details areas that need development and where actions 


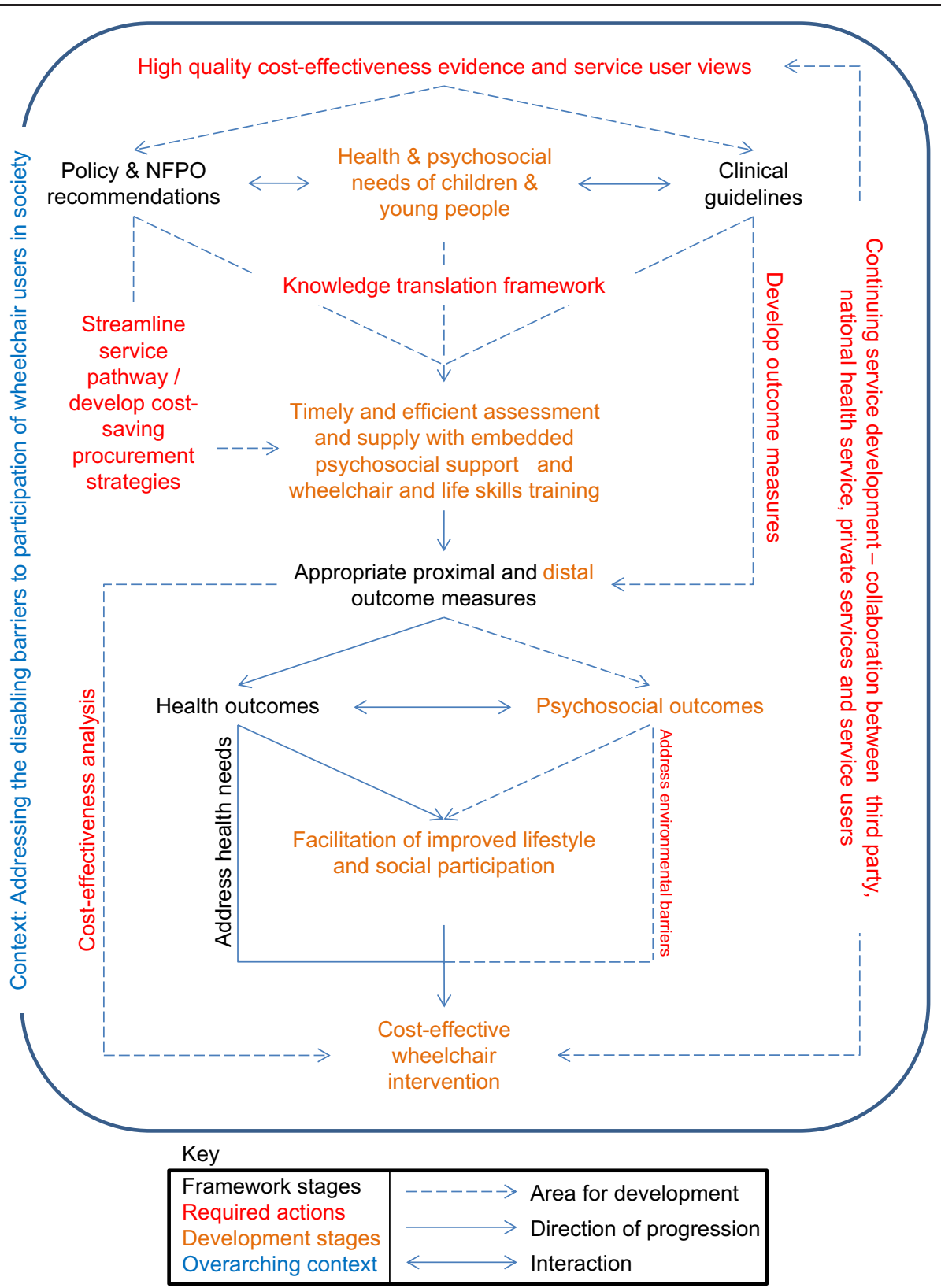

Figure 4 Conceptual framework for developing cost-effective wheelchair services for children and young people. A conceptual framework was developed from the overarching synthesis of evidence. It details areas for development and required actions in order to develop cost-effective wheelchair services.

for improving both the effectiveness and cost-effectiveness of wheelchair services for children and young people are required.

Areas for future development include: conducting and making available high quality effectiveness, cost-effectiveness and qualitative evidence; developing a knowledge translation framework; streamlining management and procurement strategies; developing appropriate outcome measures; addressing environmental barriers to wheelchair use; conducting robust cost-effectiveness analyses; and ensuring continued service development with collaboration between third party, NHS, private services and service users (children/young people and their families).

\section{Discussion}

The major contribution to knowledge from this novel mixed-method review comes from the synthesis of diverse evidence to form a new conceptual framework and 
programme theory for optimal wheelchair service provision for children.

Within this overall context, the most important finding is that for children and young people wheelchairs offer more than mobility; they offer enhanced independence, social integration and participation in age-appropriate activities. It is therefore paramount that wheelchair interventions are seen as facilitators to a new way of life. Nonetheless, disabled children and parents can find the transition to wheelchair use a traumatic process that is not yet sufficiently understood. Being able to individually tailor support for children and parents, and being able to measure these wider lifestyle benefits, is therefore a priority. Further research is needed to address these significant gaps in current knowledge.

UK policy and NFPO recommendations are reflective of the perspectives of young wheelchair users and their families, but there is a lack of effective translation of policy and evidence into practice. Although policy recommendations do correlate with the opinion evidence, the barriers to effective provision and use of wheelchairs have continued to prevail in UK NHS services over many years [11-14]. The key to improving outcomes for children and young people lies in improving service delivery, organisation and translation of knowledge of what works and what children desire from their wheelchairs.

The translation of evidence and knowledge into practice is not simply a case of publishing guidelines and policy. Evidence based practice requires specific action and commitment from services, for instance through the implementation of a knowledge translation framework such as the Knowledge to Action Process [53]. This knowledge translation process recognises the importance of gathering and synthesising evidence in a robust and replicable manner, and emphasises use of appropriate dissemination techniques and effective exchange of knowledge between researchers and knowledge users. This process is particularly useful for areas where research may be lacking, and encourages the synthesis of evidence through systematic reviews and meta-analyses in order to gather and build upon the current knowledge base [53].

Translation of evidence into practice is mitigated by the level of evidence, the context, the presence of facilitation and the success of implementation (assessing organisational outcomes and achievements) [54]. Services must therefore make a commitment to implementing a framework that promotes the translation of evidence into practice. Without specific commitment to change, services are unlikely to be developed in a way that promotes and facilitates positive change in-line with service user needs and evidence of effectiveness.

New tools have been produced to facilitate adoption of evidence into practice that could help identify the problems of evidence translation in local contexts. For example, The NHS Institute for Innovation and Improvement [55] developed the 'Spread \& Adoption' tool to aid in the assessment of innovation implementation likelihood. The purpose of this tool is to highlight small changes that can be made to promote change and ensure that an organisation is ready to implement new ideas. With the use of tools such as this, organisations can prioritise factors that require action and determine barriers to change and innovation.

The 'Any Qualified Provider' principle concerns the tendering process whereby any qualified provider can compete for NHS contracts that are awarded by the Department of Health in England. Approved providers may include state/private hospitals, charities, private organisations and certain retailers (e.g. private wheelchair suppliers). This allows patients to make informed decisions about their healthcare based on service attributes important to them, for instance how geographically close a service is or the quality of care provided. This principle promotes services that are developed around the holistic needs of the service user [56] as they can seek the most appropriate provider for their needs. It has potential for wider application if more evidence of effect is available to help inform decisions. Focussing on integrating agencies to provide better care and services for disabled children is also of paramount importance. Wheelchair services need to think outside the health domain and consider the wider needs of disabled children to ensure they are not excluded from education and social settings.

There is a distinct lack of high-quality effectiveness and cost-effectiveness evidence within this field. Although many studies have used robust methods to explore biomechanical impacts of mobility interventions (which were not relevant for this review), these do not reflect the outcomes of services from a carer or service user perspective. The intervention evidence, although limited by quality, demonstrates that wheelchair interventions may have a range of positive effects beyond mobility. However these results should be viewed with caution due to the limited quality of evidence. More evidence is required to understand how effective interventions can be measured and achieved for all service users. This requires studies to use large sample sizes, robust methods and diverse outcome measures.

The application of health economics could enable a better understanding of the cost-effectiveness of wheelchair interventions, and thus benefit service-commissioning and funding allocation, and enable these practices to be evidence-based and equitable. The limited economic evidence in this review may be considered best evidence in the field due to the lack of other research into the cost-effectiveness of wheelchairs for children and young people. 
Future research should focus on developing more appropriate outcome measures, health economic methods, and exploring the use of quality of life or capability measures to determine effectiveness from a more holistic perspective. Current wheelchair service outcome measures focus on clinical outcomes and service quality (e.g. QUEST [57]), which do not reflect all of the needs of service users. Incorporation of generic preference-based measures into routine data collection would also allow local and international collection of utility data. This could in turn be used to develop cost per QALY estimates and utility changes facilitated by wheelchair interventions. Furthermore, this evidence would allow comparisons with other healthcare interventions and understanding of incremental cost-effectiveness. This would in turn encourage appropriate funding allocation and provision based on robust effectiveness evidence.

Designing high-quality research in this field has specific challenges, particularly if looking generally at wheelchair interventions across a range of disabilities. Mobility impairment can be as a result of many different conditions, and thus needs and interventions can be highly variable. This has implications for conducting large scale trials using clinical outcomes. Likewise, interventions are likely to be highly variable across different conditions. Healthrelated quality of life and capability measures would allow a universal outcome that reflects the wider benefits of such interventions, and therefore would be a more appropriate approach to understanding the effectiveness of various interventions.

Although the use of QALYs can be contentious [58], it provides a universal measure that can compare the effectiveness of disparate interventions. For instance, different types of wheelchairs for different types of disabilities could be compared using a single outcome (QALY gains). This data could be collected alongside clinical outcomes in order to encourage holistic interventions that fit in with the needs and desires of young wheelchair users.

At present child and parent proxy versions of validated health-related quality of life measures do exist, for instance the Health Utilities Index (HUI) [59]. However, their relevance for wheelchair users is still to be demonstrated. Some measures, such as the PedsQL, have additional bolt-on questions for particular conditions (such as cerebral palsy) which take into account the conditionspecific aspects of quality of life [60].

If wheelchair services in the UK and internationally were to adopt a single set of outcome measures a wealth of data could be generated, which could be used to evaluate the holistic effectiveness of wheelchair interventions for children and young people. This data could be used to aid the development, supply and maintenance of wheelchairs. It would promote interventions that reflect the desires of service users and would allow outcomes to be measured appropriately from the perspective of the service user and the clinician. Furthermore, services could be structured around the needs of service users.

Within a UK healthcare system context, the findings provide impetus for NICE to consider wheelchair services (both adult and child) a high priority. NICE provides national clinical guidelines on healthcare interventions, medication and new health technologies in order to ensure high quality and evidence based care for patients within the NHS [61]. To date NICE have produced little guidance on disability interventions.

\section{Review limitations}

No major deviations from the protocol were noted. In the spirit of transparency, it is worth considering some potential limitations. The original aim was to understand wheelchair interventions more generally, however due to the general focus in the literature on PWC interventions, the findings have greater relevance to PWC. Over half of the intervention studies looked specifically at children with cerebral palsy. Furthermore, the intervention evidence was of low quality and at risk of bias, thus the findings must be viewed with caution.

Although evidence included in this review may not be universally generalizable to all conditions, it still offers a better understanding of what benefits are afforded by wheelchair provision. More research may be needed to see if particular benefits from wheelchairs are universal across conditions.

The lack of economic evidence highlights the issues of applying health economics to wheelchair provision for disabled children and justifies further research within this field. The lack of RCTs in this field highlights the ethical and methodological issues of wheelchair intervention studies in children. However, the study by Jones et al. [23] establishes that an RCT can be a useful and ethically sound approach when conducted appropriately. For instance, it is unethical to withhold wheelchairs from those that require them, thus standard issue wheelchairs could be used in the control group and more technologically advanced equipment in the intervention group. Likewise research examining manual versus powered wheelchairs could utilise a similar RCT setup.

Only evidence written or translated into English was included in this review, which may have excluded valuable research written in other languages.

\section{Conclusions}

Wheelchairs offer varied benefits to disabled children in terms of health, development and social inclusion. At present NHS wheelchair services in the UK are not meeting all of children's needs and service development is required. 
Findings derived from the evidence are relevant for NHS services and have some implications for wheelchair services globally. Wheelchair services have an invaluable role in promoting equality for disabled people. If these services can address disabling barriers for children at a young age, they may be able to facilitate more inclusion in education and society.

There are important gaps in current knowledge regarding health economic methods and available outcome measures, which hinder further service development and research. Health economics has an important role in developing effective, efficient and equitable wheelchair services globally. The lack of economic evidence in this field highlights the lack of appropriate methods to measure cost-effectiveness. Establishing the cost-effectiveness of interventions is a priority to promote efficient services.

Collaboration between countries on future research would allow the collection of a wealth of data regarding intervention effectiveness and cost-effectiveness. The use of universal and validated outcome measures across countries would have a distinct impact on the development of wheelchair services that promote social inclusion and independence.

\section{Additional files}

Additional file 1: Included studies and reports. Full list of included studies and reports, with details of study design, objectives, hypotheses, outcomes, measures, sample size, country, key findings and statistical evidence (where appropriate).

Additional file 2: Quality appraisal tools and outcomes. Full list of quality appraisal tools and quality appraisal outcomes for each intervention, opinion and economic evidence study.

\section{Competing interests}

The authors have no financial or non-financial competing interests to declare.

\section{Authors' contributions}

$\mathrm{NB}, \mathrm{JN}, \mathrm{RTE}$ and $\mathrm{NH}$ were responsible for the review conception and design and the development of the original review protocols. All authors were involved in reading and approving the manuscript. NB conducted the searches, quality appraisal, data extraction processes, analyses of evidence and synthesis of evidence, with support from JN, RTE and NH. JN provided evidence synthesis expertise and supervisory support.

\section{Authors' information}

NB is a Health Economics PhD student at Bangor University, supervised by RTE, JN and NH. RTE is Professor of Health Economics at Bangor University. She has expertise in the economic evaluation alongside trials of complex public health and psychosocial interventions, and an interest in the health economics of disability. JN has experience in child health research, health services research with embedded health economics and evidence synthesis. $\mathrm{NH}$ is Director of the Bath Institute of Medical Engineering (Designability) and a visiting Professor from University of Bath. His recent research projects include the use of assistive technology to support home based rehabilitation following stroke and monitoring patterns of physical activity during rehabilitation.

\section{Acknowledgements}

We would like to acknowledge the support of Dr. Meena Mishra for assisting in the screening and appraising of evidence, Marion Poulton for providing input in the development of the search strategy, and Emma Bray for assisting in editing and proofreading the manuscript. The systematic review was funded by the National Institute for Social Care and Health Research as part of a PhD studentship award. The systematic review protocol was registered with PROSPERO (http://www.crd.york.ac.uk/PROSPERO/).

\section{Author details}

${ }^{1}$ Centre for Health Economics and Medicines Evaluation, Bangor University, Ardudwy Building, Normal Site, Bangor University, Bangor, Gwynedd LL57 2PZ, UK. ${ }^{2}$ Centre for Health-Related Research, Fron Heulog, Bangor University, Bangor, Gwynedd LL57 2EF, UK. ${ }^{3}$ DesignAbility, Bath Institute of Medical Engineering, The Wolfson Centre, Royal United Hospital, Bath BA1 3NG, UK.

Received: 6 February 2013 Accepted: 11 July 2014

Published: 17 July 2014

\section{References}

1. World Health Organisation: Concept Note. World Report on Disability and Rehabilitation. Geneva, World Health Organization. Geneva: World Health Organization; 2008 [http://www.who.int/disabilities/publications/dar_ world_report_concept_note.pdf]

2. World Health Organization: World Report on Disability. Geneva: World Health Organization; 2011 [http://whqlibdoc.who.int/publications/2011/97892406 85215_eng.pdf]

3. Sheldon S, Jacobs NA (Eds): Report of a Consensus Conference on Wheelchairs for Developing Countries, Bangalore, India, 6-11 November 2006 Copenhagen: International Society for Prosthetics and Orthotics; 2007 [http://pdf.usaid.gov/pdf_docs/PNADP827.pdf]

4. World Health Organization: Guidelines on the Provision of Manual Wheelchairs in Less-Resourced Settings. Geneva: World Health Organization; 2008 [http://www.who.int/disabilities/publications/technology/ wheelchairguidelines/en/index.html]

5. United Nations: The Standard Rules on the Equalization of Opportunities for Persons with Disabilities. Preconditions for Equal Participation. 1993 [http://www.un.org/esa/socdev/enable/dissre03.htm]

6. World Health Organization: The Global Burden of Disease: 2004 Update. Geneva: World Health Organization; 2008 [http://www.who.int/healthinfo/ global_burden_disease/GBD_report_2004update_full.pdf]

7. Lau H, Tam EWC, Cheng JCY: An experience on wheelchair bank management. Disabil Rehabil Assist Technol 2008, 3(6):302-308.

8. Welsh Assembly Government: National Service Framework for Children, Young People and Maternity Services in Wales. Cardiff: Welsh Assembly Government; 2005. 
9. Department of Health Commissioning Team: Local Innovation in Wheelchair and Seating Services. London: Department of Health; 2010.

10. Contact a Family: Annual Review: How we Made a Difference in 2010/2011. London: Contact a Family; 2011 [http://www.cafamily.org.uk/media/447274/ contact_a_family_annual_review_2010-11a.pdf]

11. National Assembly for Wales Health, Wellbeing and Local Government Committee: Report on Inquiry into Wheelchair Services in Wales. Cardiff: National Assembly for Wales; 2010.

12. Audit Commission: Fully Equipped 2002- Assisting Independence. Wetherby: Audit Commission Publications; 2002.

13. NHS Modernisation Agency: Improving Services for Wheelchair Users and Carers-Good Practice Guide. London: Department of Health; 2005.

14. Prime Minister's Strategy Unit Report: Improving the Life Chances of Disabled People.' Joint Report With Department for Work and Pensions, Health, Education and Skills and the Office of the Deputy Prime Minister. London: Department for Work and Pensions, Health, Education and Skills; 2005 [http://webarchive.nationalarchives.gov.uk/+/http:/www.cabinetoffice.gov. uk/media/cabinetoffice/strategy/assets/disability.pdf]

15. Department of Health: Standard 8 of the Children's National Service Framework: Disabled Children and Those With Complex Healthcare Needs. London: Department of Health; 2004.

16. Oliver M: Theories of disability in health practice and research. BMJ 1998, 317:1446-1449.

17. Centre for Reviews and Dissemination: Systematic Reviews: CRD's Guidance for Undertaking Reviews in Health Care. York: University of York; 2009.

18. Thomas J, Harden A: Methods for the thematic synthesis of qualitative research in systematic reviews. BMC Med Res Methodol 2008, 8:45.

19. Oliver $S$, Harden $A$, Rees $R$ : An emerging framework for including different types of evidence in systematic reviews for public policy. Evaluation 2005, 11(4):428-446.

20. EPPI-Centre: EPPI-centre methods for conducting systematic reviews. 2010, [http://eppi.ioe.ac.uk/cms/LinkClick.aspx?fileticket=hQBu8y4uVwl\% $3 \mathrm{~d} \&$ tabid $=88 \&$ mid $=6162]$

21. Jones MA, McEwen IR, Hansen L: Use of power mobility for a young child with spinal muscular atrophy. Phys Ther 2003, 83(3):253-262.

22. Furumasu J, Tefft D, Guerette P: The Impact of Early Powered Mobility on Young children's Play and Psychosocial Skills. In Proceedings of 24th International Seating Symposium, March 6-8, 2008; Vancouver. 2008:160-165

23. Jones MA, McEwan IR, Neas BR: Effects of Power Wheelchairs on the development and function of young children with severe motor impairment. Pediatr Phys Ther 2012, 24(2):131-140.

24. Tefft D, Guerette P, Furumasu J: The impact of early powered mobility on parental stress, negative emotions, and family social interactions. Phys Occup Ther Pediatr 2011, 31(1):4-15.

25. Bottos M, Bolcati C, Sciuto L, Ruggeri C, Feliciangeli A: Powered wheelchairs and independence in young children with tetraplegia. Dev Med Child Neurol 2001, 43(11):769-777.

26. Deitz J, Swinth Y, White O: Powered mobility and preschoolers with complex developmental delays. Am J Occup Ther 2002, 56(1):86-96.

27. Huhn K, Guarrera-Bowlby P, Deutsch JE: The clinical decision-making process of prescribing power mobility for a child with cerebral palsy. Pediatr Phys Ther 2007, 19(3):254-260.

28. Meiser MJ, McEwen IR: Lightweight and ultralight wheelchairs: propulsion and preferences of two young children with spina bifida. Pediatr Phys Ther 2007, 19(3):245-253.

29. Østensjø S, Carlberg EB, Vøllestad NK: The use and impact of assistive devices and other environmental modifications on everyday activities and care in young children with cerebral palsy. Disabil Rehabil 2005, 27(14):849-861.

30. Benedict RE, Lee JP, Marrujo SK, Farel AM: Assistive devices as an early childhood intervention: evaluating outcomes. Technol Disabil 1999, 11(1):79-90.

31. Wiart L, Darrah J, Hollis V, Cook A, May L: Mothers' perceptions of their children's use of powered mobility. Phys Occup Ther Pediatr 2004, 24(4):3-21.

32. Durkin J: Discovering powered mobility skills with children: 'responsive partners' in learning. Int J Ther Rehabil 2009, 16(6):331-341.

33. Guerette P, Tefft D, Furumasu J: Pediatric powered wheelchairs: results of a national survey of providers. Assist Technol 2005, 17(2):144-158.
34. Home AM, Ham R: Provision of powered mobility equipment to young children: the Whizz-Kidz experience. Int J Ther Rehabil 2003, 10(11):511-519.

35. Staincliffe $\mathrm{S}$ : Wheelchair services and providers: discriminating against disabled children? Int J Ther Rehabil 2003, 10(4):151-158.

36. Wiart L, Darrah J, Cook A, Hollis V, May L: Evaluation of powered mobility use in home and community environments. Phys Occup Ther Pediatr 2003, 23(2):59-75.

37. Evans S, Neophytou C, De Souza L, Frank AO: Young people's experiences using electric powered indoor-outdoor wheelchairs (EPIOCs): Potential for enhancing users' development? Disabil Rehabil 2007, 29(16):1281-1294.

38. Lawlor K, Mihaylov S, Welsh B, Jarvis S, Colver A: A qualitative study of the physical, social and attitudinal environments influencing the participation of children with cerebral palsy in northeast England. Pediatr Rehabil 2006, 9(3):219-228.

39. Shahid M: Buggy-to-wheelchair progression for children with cerebral palsy: parents' and therapists' opinions. Int J Ther Rehabil 2004, 11(12):560-566.

40. Curtin M, Clarke G: Listening to young people with physical disabilities' experiences of education. Int J Disabil Dev Educ 2005, 52(3):195-214.

41. Muscular Dystrophy Campaign: Building on Foundations: Get Moving - the Case for Effective Wheelchair Services. London: Muscular Dystrophy Campaign; 2010

42. Muscular Dystrophy Campaign: Wheelchair Provision for Children and Adults With Muscular Dystrophy and Other Neuromuscular Conditions. London: Muscular Dystrophy Campaign; 2011.

43. Barnardos and Whizz-Kidz: Don't Push us Around. London: Whizz-Kidz; 2006

44. Care Services Improvement Partnership: Out and About- Wheelchairs as Part of a Whole-Systems Approach to Independence. London: Department of Health; 2006.

45. HM Treasury and Department for Education and Skills: Aiming High for Disabled Children: Better Support for Families. London/Runcorn: HM Treasury/ Department for Education and Skills; 2007.

46. Scottish Executive: Moving Forward: Review of NHS Seating Services in Scotland. Edinburgh: Scottish Executive; 2006.

47. All Party Parliamentary Group for Paediatric Reform and Whizz-Kidz: "My Wheelchair is my Shoes" Making the Case for Wheelchair Reform. London: Whizz-Kidz; 2011.

48. Neilson A, Hogg J, Malek M, Rowley D: Impact of surgical and orthotic intervention on the quality of life of people with profound intellectual and multiple disabilities and their carers. J Appl Res Intellect Disabil 2000, 13:216-238.

49. Curtis L, Netten A: Unit Costs of Health and Social Care 2006. Cantebury: Personal Social Services Research Unit, University of Kent; 2006 [http://www. pssru.ac.uk/archive/pdf/uc/uc2006/uc2006.pdf (last accessed 24/07/2012]

50. Curtis L: Unit Costs of Health and Social Care 2011. Cantebury: Personal Social Services Research Unit, University of Kent; 2011 [http://www.pssru.ac. uk/pdf/uc/uc2011/uc2011.pdf]

51. Frontier Economics: Impact of Whizz-Kidz Support to Primary Care Trusts. London: Frontier Economic Ltd; 2011.

52. Professor the Lord Darzi of Denham KBE: High Quality Care for all: NHS Next Stage Review Final Report. London: Department of Health; 2008.

53. Graham ID, Tetroe JM: Getting evidence into policy and practice: perspective of a health research funder. J Can Acad Child Adolesc Psychiatry 2009, 18(1):46-50.

54. Stetler CB, Damschroder $\sqcup$, Helfrich CD, Hagedorn HJ: A Guide for applying a revised version of the PARIHS framework for implementation. Implement Sci 2011, 6:99.

55. NHS Institute for innovation and Improvement: Spread and adoption tool. 2012, [http://www.institute.nhs.uk/index.php?option=com_spread_and_ adoption]

56. Posture and Mobility Group: The Future of Wheelchair Services - Any Qualified Provider?. Exeter: Posture and Mobility Group and British Healthcare Trades Association; 2011 [http://www.pmguk.co.uk/official-publications-and-reports. html]

57. Demers L, Weiss-Lambrou R, Ska B: The Quebec User Evaluation of Satisfaction with Assistive Technology (QUEST 2.0): An overview and recent progress. Technol Disabil 2002, 14:101-105 [https://enablemob.wustl. edu/OT572D-01/RequiredArticles/QUEST\%20article.pdf]

58. Nord E, Daniels N, Kamlet M: QALYs: some challenges. Value Health 2009, 12:1 S10-S15 [http://www.ispor.org/meetings/invitational/QALY/Paper3.pdf] 
59. Horsman J, Furlong W, Feeny D, Torrance G: The Health Utilities Index (HUI): concepts, measurement properties and applications. Health Qual Life Outcomes 2003, 1(54) [http://www.hqlo.com/content/1/1/54]

60. Varni JW, Burwinkle TM, Seid M: The PedsQL as a pediatric patientreported outcome. Reliability and validity of the PedsQL measurement model in 25,000 children. Expert Rev Pharmacoecon Outcomes Res 2005, 5(6):705-719.

61. National Institute for Health and Clinical Excellence: The guidelines manual. [http://publications.nice.org.uk/the-guidelines-manual-pmg6]

doi:10.1186/1472-6963-14-309

Cite this article as: Bray et al:: Wheelchair interventions, services and

provision for disabled children: a mixed-method systematic review and conceptual framework. BMC Health Services Research 2014 14:309.

\section{Submit your next manuscript to BioMed Central and take full advantage of:}

- Convenient online submission

- Thorough peer review

- No space constraints or color figure charges

- Immediate publication on acceptance

- Inclusion in PubMed, CAS, Scopus and Google Scholar

- Research which is freely available for redistribution 